Note: This is a pre-publication final draft. For the published version, please see: Rafalow, Matthew H. 2016 "Tinkering Online: Digital Supports for Making and Sharing." In Makeology: Makerspaces as Learning Environments, edited by Kylie Peppler, Erica Halverson, and Yasmin Kafai. New York: Routledge Press.

\title{
Tinkering Online: Digital Supports for Making and Sharing
}

\author{
Matthew H. Rafalow \\ University of California-Irvine
}

I had a friend who just didn't understand what I meant ... and she said "I wish you lived closer so I could SEE you do it. lightbulb! And now, I have videos of basic stitches on my blog. My blog is really just a way to share my projects with family and friends that don't use ravelry. lol (Jen2291, Hogwarts at Ravelry participant)

Studies of making and maker culture are increasingly looking towards online environments as a nexus for learning and creative production. On the one hand, scholars have expanded the types of products that constitute made goods to include not only tangible objects, like knits and electronics, but also new media, such as fan fiction, digital graphics, and computer programs (Eisenberg and Buechley 2008; Guzzetti et al. 2010; Lankshear and Knobel 2010; Spencer 2005; Wortham 2011). On the other, research on digital media and learning finds that online communities are fruitful spaces for learners to develop the skills needed to pursue making activities, particularly for new media-related endeavors (Ito et al. 2009; 2013; Korobkova 2014; Kow et al. 2014; Martin 2014; Pfister 2014; Rafalow and Tekinbas 2014).

Little work, however, explores how online learning environments operate not only as standalone communities of practice but also how they can be meaningfully tethered to other parts of learners' lives, both online and offline. Connected learning theory posits that "openly networked design" provides multiple avenues for learners to connect their online activities with other spheres of their lives (Ito et al. 2013). For example, through her work as a participant of Hogwarts at Ravelry, an online community centered around an interest in knit construction, user Jen2291 (49 years old) created a how-to blog that extends her work from the online space to her friends and family that are not part of the community. In this way, learners take advantage of the networked design of digital platforms to connect their making experiences across a broader learning ecology, or the multifaceted character of environments where intergenerational audiences of youth and young adults learn, develop, and grow (Weisner 2002).

This chapter builds on these themes by illustrating how intergenerational audiences that include youth and young adults pursue learning experiences in maker communities online and use openly networked design features to connect making practices to other parts of the learning ecology. Like Jen2291, I find that participants of these online communities develop skills to tinker online and produce various made goods, like video game levels and knits, and then connect these making activities out to others outside of the community like family and friends. To comment on these points, I 
first describe the structure of online platforms that inform connected participation in two distinct cases: a gaming community centered on level design (Sackboy Planet), and a Harry Potter-themed knitting community (Hogwarts at Ravelry). While Sackboy Planet participants devote their efforts towards new media products, Hogwarts at Ravelry members develop both digital and physical materials as part of their pursuits. Both cases thus reveal interesting comparisons for online and offline maker activities. I then document how various participants tinker online and take advantage of openly networked design to connect making experiences to other parts of their learning ecology, including online and offline networks of family and friends.

\section{Connected Learning, Digital Youth and Making}

Connected learning is a growing research movement in both education and tech sectors that aims to understand new supports for learning in an increasingly networked society. Its proponents study how the Internet has opened new opportunities for learning and creative production online (Ito et al. 2013). Work on connected learning has considerable overlap with research on making and makerspaces given that both sets of literature explore the critical role that making plays in development literacies for the $21^{\text {st }}$ century. These literacies consider not only learning skills necessary for the production of tangible crafts or print writing but also new media-rich digital creations such as blogging, video production, and computer programming (Gee 2010).

Connected learning theory stands on the shoulders of research around the role of media in consumption and production practices in contemporary society. Jenkins (2006) describes the contemporary media landscape as a "convergence culture" where multiple genres of media overlap and pave the way for new forms of engagement. For example, Black (2008) documents how youth create and share extensive writing projects through their interest in fan fiction, blending together media from popular cultural interests with writing on new media platforms online. In another example, Ito et al. (2012) illustrates how youth pursue their interest in anime music videos by creating "machinima," or new video creations produced by remixing and editing other videos available online, and develop important new literacies in media production through a convergence of media platforms and tools. The online communities where these learners hang out and improve their craft reflect the increasing prevalence of a "participatory culture" enabled by how participants adopt new media platforms online to pursue collaborative learning experiences (Jenkins 2006). This body of work thus illustrates the potential for online environments, including web-based forums, video-sharing communities, and even online gaming platforms to embody a community of practice centered on knowledge sharing and making (Gee 2003).

Youth and young adults are poised to take advantage of participatory cultures online as a means to develop their skills and improve their craft as creators and makers. Through a multi-case study of youth pursuits online, Ito et al. (2009) finds that youth cultural pursuits online occupy different genres of engagement that can overlap to drive learning and making. First, youth extend their peer cultures online through friendshipdriven participation, and hang out in chatrooms, blogs, forums, and other participatory platforms to develop connections and a shared culture. Second, some youth pursue interest-driven activities online as they discover communities of practice organized 
around their favorite fan and media cultures. These youth can "mess around," or tinker, with new tools for making, and "geek out," or engage with expertise-driven forms of participation around supportive peers and mentors. This work thus illustrates the capacity for youth to pursue making online even when local contexts may not provide such infrastructure.

Although this scholarship on digital youth and making identify the importance of connecting learning activities to multiple spheres of young people's lives, the literature offers few examples of how this works in practice (Kow et al. 2014; Korobkova 2014; Martin 2014; Rafalow and Tekinbas 2014). Identifying these moments are important because they illustrate how learning can be linked to others as part of a broader learner ecology. This ecology reflects that children are situated in assorted practices as they move through institutions and develop relationships with families, peers, and neighborhoods (Barron 2006; Bronfbrenner 1979; Horst et al. 2010). The learning ecology thus positions making projects as not simply located in one isolated classroom or online community, but rather as productively connected to other aspects of the lives of youth.

I build on this literature by illustrating how connected learning communities online provide meaningful learning and making opportunities, but that in addition youth connect these practices to other contexts as well, both online and offline. Moments of transference are a necessary feature of not only the development of $21^{\text {st }}$ century literacies but its productive deployment across domains that links multiple sites of learning that achieves "network effects" where more value is created when the number and diversity of participants is increased (Ito et al. 2009; Liebowitz and Margolis 1994).

\section{Cases and Method}

To explore the relationship between making and online supports, I selected two online communities for study that illustrate these connections as youth and young adults pursue their interest in different making practices. The first case is Sackboy Planet, a companion online player community to the Playstation 3 video game LittleBigPlanet 2 (LBP2) created by Media Molecule. Key features of the game include opportunities for players to craft levels and planets within the galaxy of Craftworld. Central to the design of the game are a number of digital tools that give players the means to create their own levels, animations, character art, and soundtracks (see Figure 1 for sample image of level editor). Sackboy Planet, has more than 1,000 active members (23,000 have registered since its launch several years ago). LittleBigPlanet 2 and its associated player-created design communities establish a shared purpose around creative production, feedback, and the development of technical expertise through their various community initiatives. 


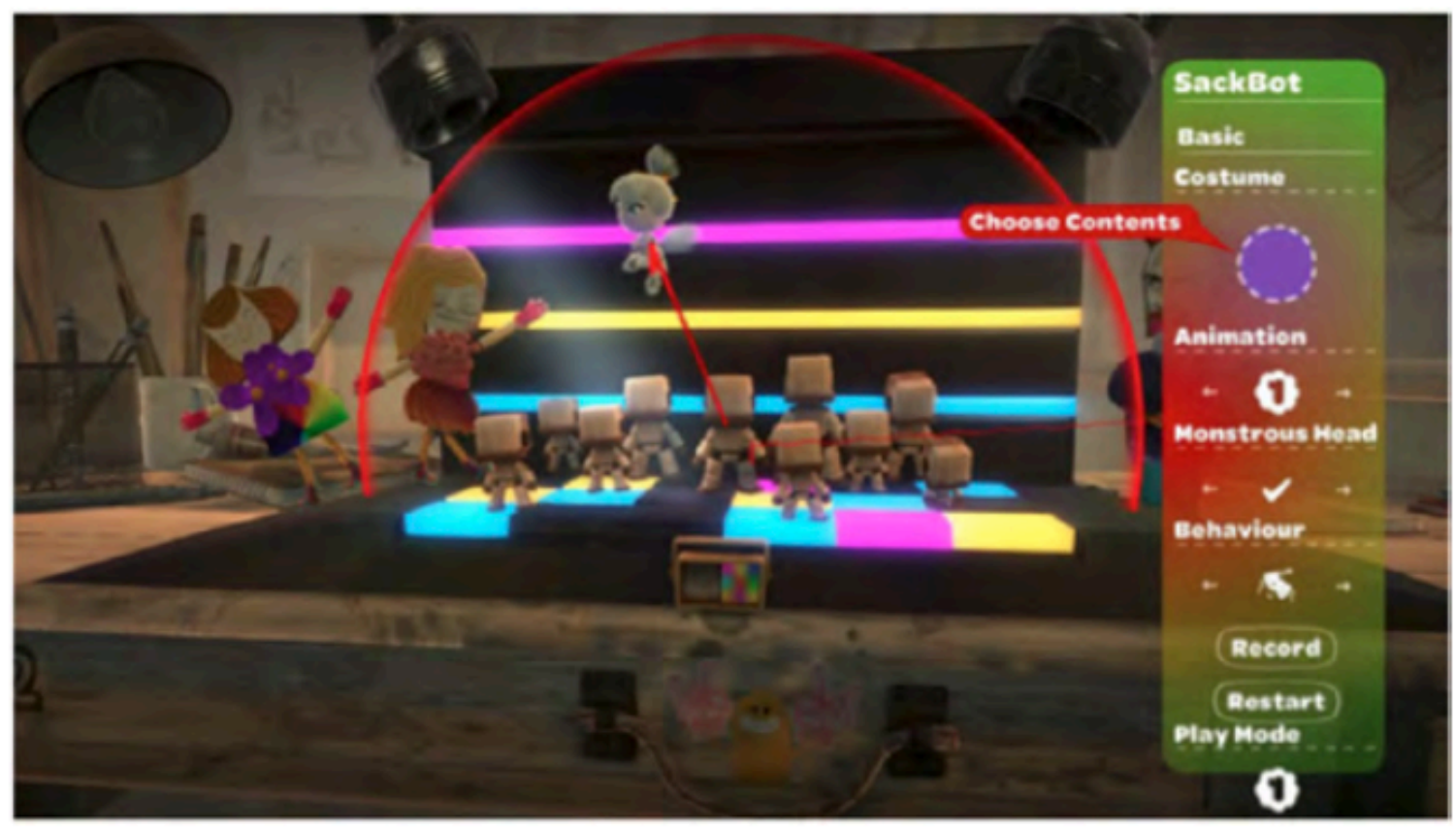

Figure 1: Screen shot of the LBP2 level editor.

The second case is Hogwarts at Ravelry, an online community centered on the shared interests of Harry Potter and fiber crafting (see Figure 2 for sample image of the website). The Harry Potter series is a popular young adult book and movie series about a boy wizard who attends the magical school of Hogwarts. Hogwarts at Ravelry, founded in 2009 by a 10-year-old girl, is one of more than 30,000 groups on Ravelry.com, which describes itself as "a community site, an organizational tool, and a yarn \& pattern database for knitters and crocheters." Ravelry.com was founded, in part, as an online social hub for fiber crafters and offers its users forums and member-created groups through which they can share their fiber crafting and other interests. 


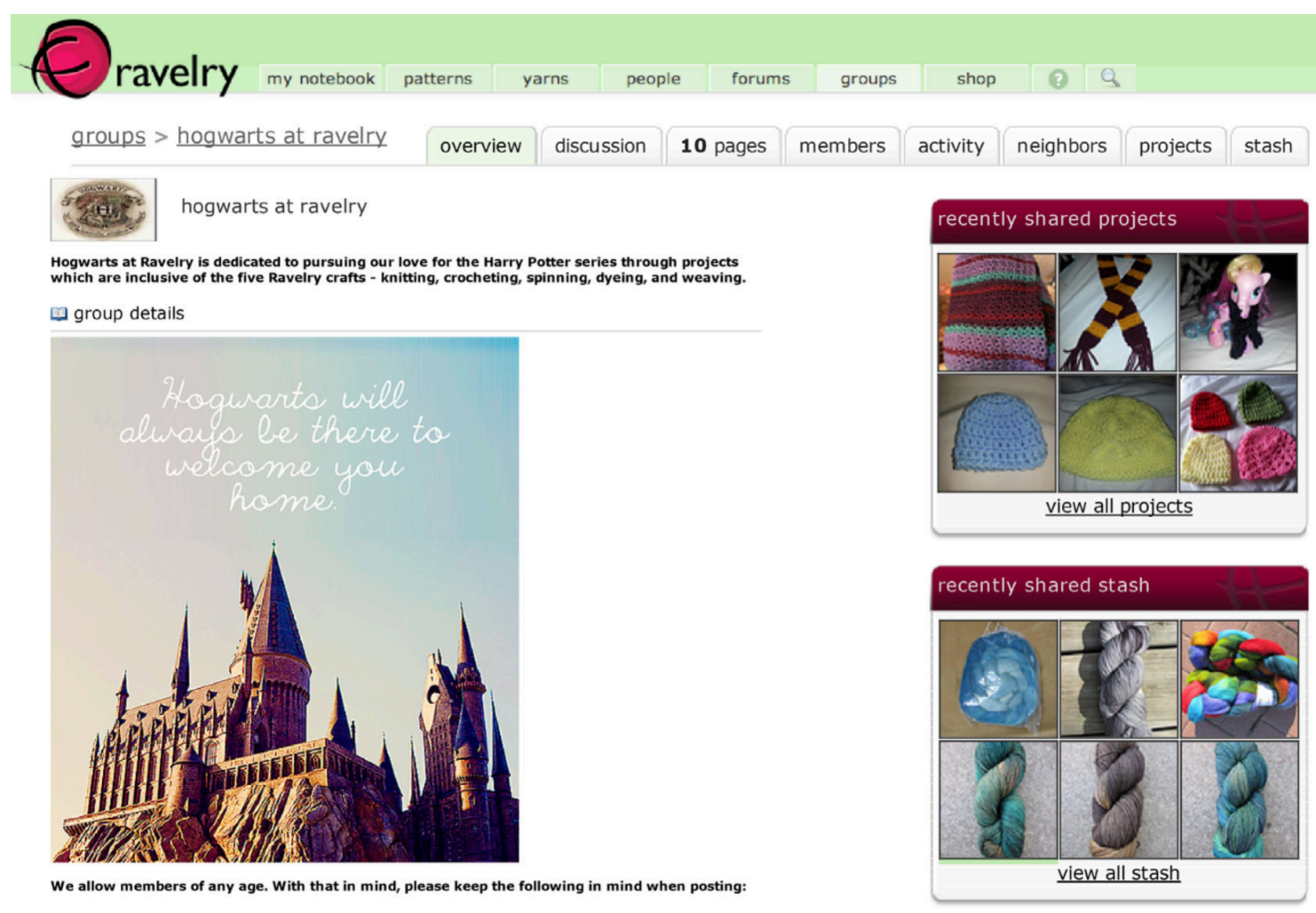

Figure 2: Screen shot of Hogwarts at Ravelry's main page. Image from Ravelry.com.

Both case studies were developed using anonymized data collected from observation- and interview-based methods. I observed Sackboy Planet for a nine-month period, documenting interaction in online forums and chatrooms, and interviewed 24 players within the community. My colleague, Rachel Pfister, observed Hogwarts at Ravelry for two years, participating in the social communities on the website, and also interviewed 24 interviews with members of the community.

In the following sections, I build from existing work on online learning by first describing the two online community platforms that structure openly networked participation; I then provide examples of ways that community members tinker online across these platforms; and I conclude by describing examples of learners from these two distinct cases who develop skills as creative producers and connect out those activities to others beyond the platform.

\section{Openly Networked Design for Making and Sharing}

The design of both the LittleBigPlanet 2 video game and the Sackboy Planet community enable openly networked sharing across various social spheres. As part of the game design, participants can not only play video games but also create and share them, too. Other players can see another individual's or group's work, comment on it, contribute to it, or even use it as a starting point for their own idea to create many opportunities for making and sharing. Certain features of the game create invitations to push people together as part of a collaborative process. For example, the game requires that there be multiple players to earn all the items in some levels, and prompts people at 
the start of gameplay to invite others to play together. These design features often lead to friendships for collaboration and learn about others' designs.

Perhaps the game's most innovative design feature is how it connects players' made goods to other networks online outside of the confines of the game itself. Through LBP.me, an online database of game information created by the game designers, information within the game can be connected outside of the game to other online publics, such as Sackboy Planet (see Figure 3 for sample image of LBP.me interface). When players begin playing LBP2, they are soon guided by the game to create their first levels. After saving their first levels, the players return to the main menu and find that the levels they just created now exist in a small region of the of each player's own world map. As they use the level editor, they are able to publish their level design through LBP.me. The game prompts players to answer a few questions, including the game's title, if it should have an associated picture, and the game's most central characteristics. These details are then added to an entry on the LBP.me database and then transmitted online. Other players can then find the player's published levels by accessing LBP.me either within the game or via the website online. Through this process, players' work becomes networked and is accessible through other online contexts, as well. Sackboy Planet is one such context where community members use LBP.me to link their own creations and embed them in interactions like forums.

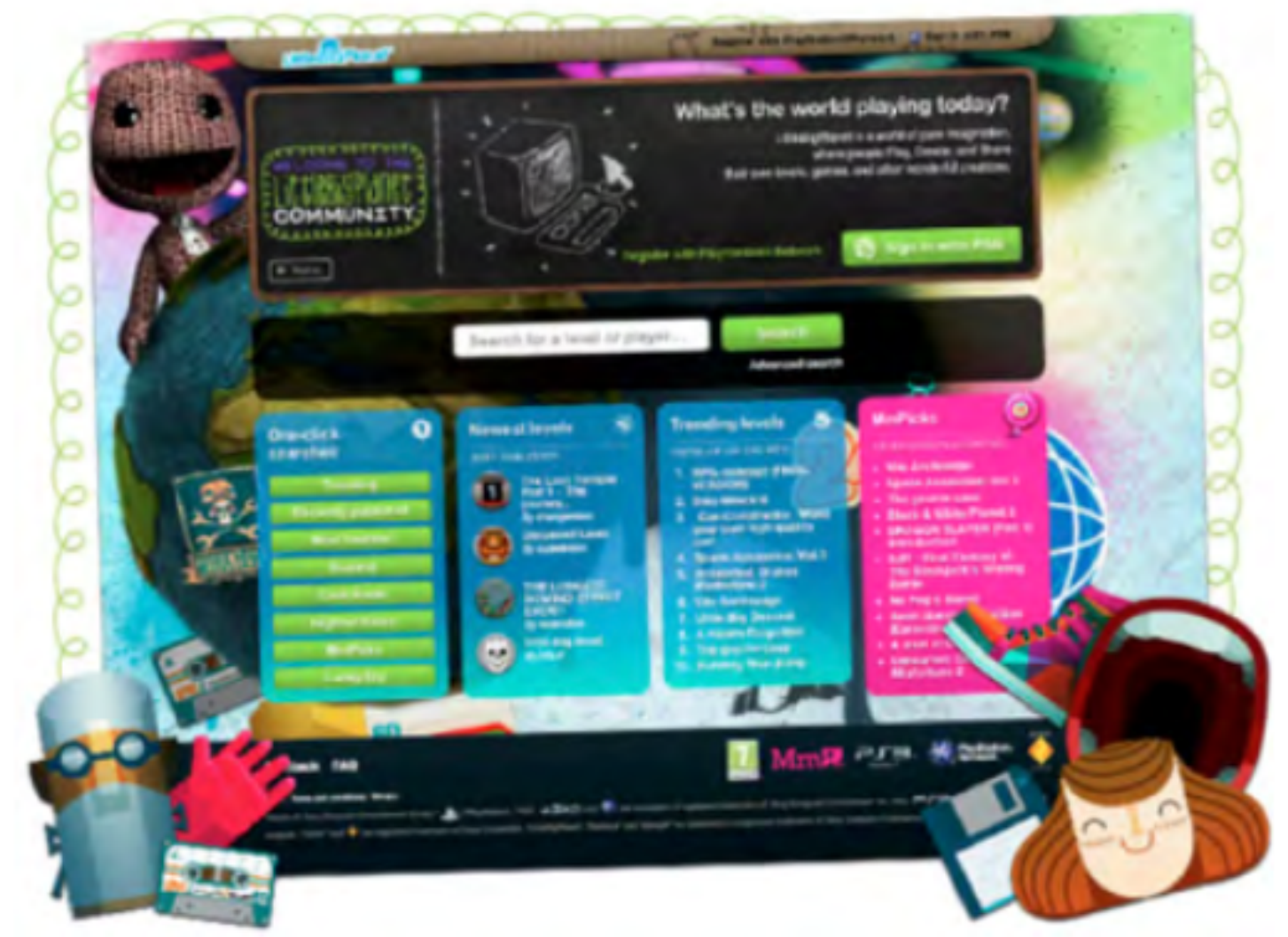

Figure 3: The LBP.me interface includes multiple ways to search and share published levels. 
Abby (21 years old) is an established player and creator in the Sackboy Planet community, and she takes advantaged of the game's openly networked design to share and embed her creations in other networks outside of the confines of the community. Abby's interests in the LBP2 design stem from her own passion for art and graphics design. As other community members report, her level designs are respected in large part to the aesthetic features she integrates into her games. Abby also maintains a Deviant Art website with many of her own fan art creations, often with illustrations in the image of characters and scenes from the LBP2 game. She met other creators not only through participation in Sackboy Planet but also through other social media networks. "I mostly watched tutorials on YouTube and took inspiration from other people's levels... [then] I made my own creations and put them out on the Internet, and people played them... it allows you to stay in touch with your fans." Players like Abby move fluidly between design within the game and other online networks, both Sackboy Planet and others, due to how they adopt the openly networked design of the platform:

LittleBigPlanet has a website called LBP.me which has everyone's profile and links to their levels... and I just put up a link to the LBP.me page of that level on my Twitter. The majority of my followers [on Twitter] are my LittleBigPlanet creator friends. I think some of them are fans of mine, as well.

Abby's participation reflects a productive interaction between convergence and participatory cultures, as well as digital supports for learning, in the interest of making new media products. But Abby's made goods, which in the context of LBP2 are in the form of designed levels with artistic flair, can be circulated across spheres beyond the confines of the Sackboy Planet maker space into other online publics like Twitter or even YouTube. Abby uses the openly networked design of the LBP2 platform to link to her creations to other networks to receive feedback, connect with other designers, and build her following.

Due to the nature of their craft, participants on Hogwarts at Ravelry exhibit many different ways to pursue making practices and share made goods across multiple spheres, both online and offline. Fiber crafts are portable and create material goods that can be worn or gifted. Often participants will wear the clothes that they knit or give away items they made for babies to friends and family. In interviews, participants describe how they will knit at church, in school, and even while out on errands. Sometimes, their on-the-go knitting attracts attention from others that provide opportunities for sharing what they have learned from online participation to others, as well:

One of the costumers [at a theater production] saw me knitting and started asking me some basic questions. After a few minutes she confessed that she was trying to figure out how to re-create a historical hat for an upcoming show. She gave me the name of the hat style, I did a little research since I was unfamiliar with it, and then gave her some recommendations on what would be the best yarns to use and what technique would create a similar look. (Tori, 23 years old, Hogwarts at Ravelry participant) 
Tori's knitting skills, in this new setting, provided an opportunity for her interest and skill set to be transposed into making practices beyond the confines of her online community and her own private face-to-face making activities.

In addition to day-to-day opportunities to share in face-to-face settings, Hogwarts at Ravelry members have access to openly networked design features of the platform that allow them to share media representations of their designs with other networks. Ravelry allows its users to create portfolios of their projects, including images of knits, which are viewable by all other participants, and render them public via a "share this" link at the top of each portfolio page. Like LBP.me, the Ravelry website operates like a database, collecting links to patterns across thousands of users' websites, blogs, and forums. Participants can also connect their own blog to the Ravelry page, which streams their entries on their profile within the Hogwarts at Ravelry community website, as well (see Figure 4 for sample image of Ravelry interface).

Some participants on the community use these features to connect their work across other online platforms, as well. For example, Jen2291 uploads a number of her patterns and resources to the system, and also creates video tutorials that she shares online. She shares links to these resources with friends online as well as family and friends offline to provide suggestions. In this way, Jen2291 moves fluidly online and offline to share making practices with different audiences: she represents her knitting work online, in the form of pictures and also instructional materials, which then connects to friends and family online so that they may engage in their own making experiences offline. Participants like Jen2291 thus distribute making practices across a broader learning ecology via the openly networked features of the platform. The links between each of these spheres are made visible as a result, and barriers to crossing them minimized, allowing members and sites to connect.

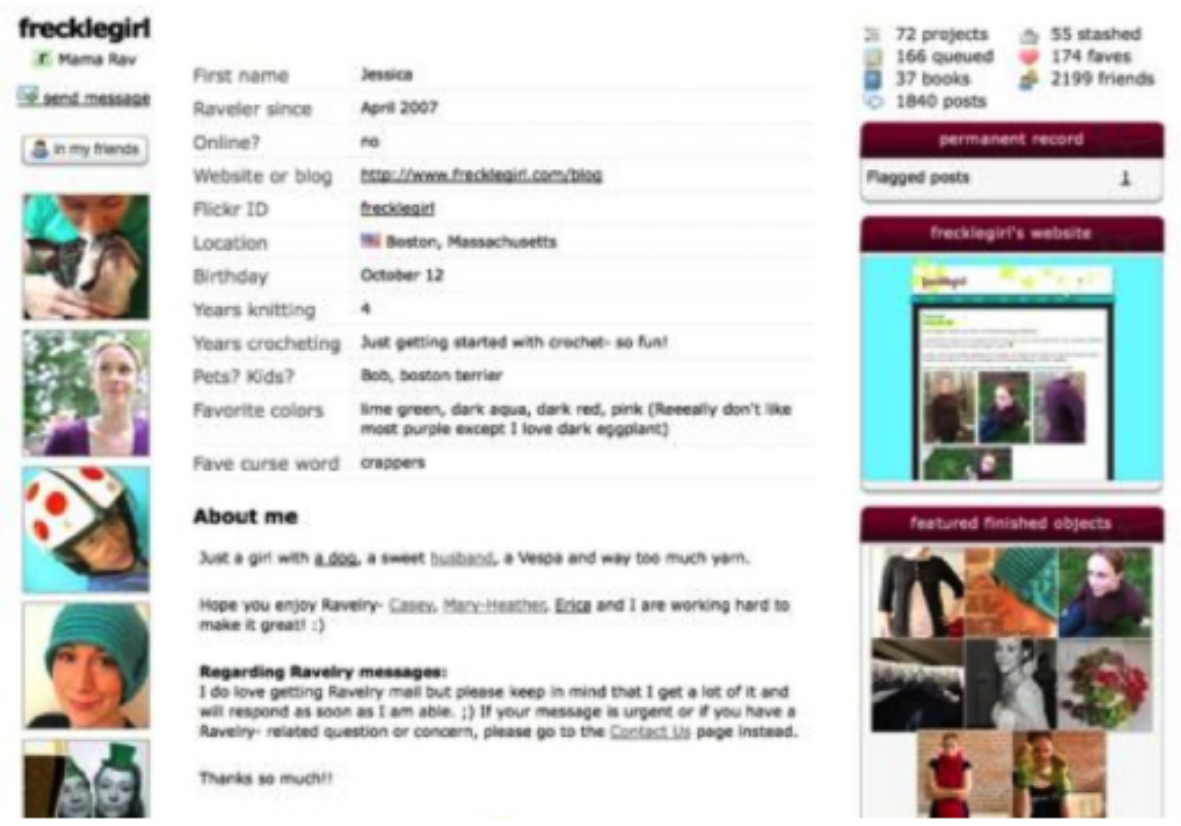

Figure 4: Sample Ravelry profile page that links participants' websites or blog with their own narrative. Image from Ravelry.com. 


\section{Tinkering Online}

Consistent with studies of digital youth, participants of these online communities engage in different types of activities that provide opportunities for "messing around," or tinkering, with various tools to "geek out," or develop proficiency as budding makers (Ito et al. 2009).

The forums and community wiki on Sackboy Planet provide many different kinds of making-related resources and activities for participants to pursue. For example, established community members curate tutorials for others to learn how to design particular features as youth figure out what to build. One participant, Sensei (27 years old), created and shared a guide that included video-based design tutorials that became a major fixture of learning in the community. One tutorial shows others how to use the tools in the level editor to incorporate probability into their designs. In the video, Sensei explains how to create a "flicker" effect with a broken lightbulb. With the design tools in the game editor, he creates randomizers that inform whether or not the light flickers, and he binds a flicker sound to the light changes in order to complete the effect. Tutorials like Sensei's are peer-created resources that others in the community use as they build their own creations.

Although tutorials are typically created by more advanced creative producers, other members of Sackboy Planet provide feedback on these tutorials to test the advice and make suggestions for improvement. Resources are rarely created by one person alone - knowledge only becomes commonplace after it has been tested and tinkered by the broader community base. In the comments of the tutorial, many of the responses are mostly appreciative in nature ("This is great!"), and some offer follow-up questions. For example, several community members noted that Sensei had an error in his probability tutorial. Another participant said that they liked the background images he used in his tutorial, which other readers helped identify for reuse in their own projects (though this was not the subject of the video). While Sensei created the video to provide specific lessons about probability in the game, users engaged with the tutorial in their own way in the comment thread to broaden knowledge shared about the game design process.

For example, one participant named Raybeam posted a question about a level he was designing and wanted to know how to calibrate gravity as part of the experience: "Does anyone have any suggestions about how to create a moving elevator that keeps the feet of the character firmly on the ground? Whenever my elevator stops at the top it launches my character off to the roof because of the gravity." Raybeam's question is essentially about tinkering with physics; he wants to create a realistic elevator, but to do so requires knowledge of design mechanics that manipulate gravity. In response, others asked questions and shared feedback to help figure out his puzzle. "Have you tried using material that sticks the character to the elevator?" "What about setting air resistance to $100 \%$ on the elevator so the character stays put?" After a back-and-forth, Raybeam shared a link to his draft level so that readers can test it and offer other suggestions. The last response provided the most conclusive suggestion: to create a sensor on the elevator with a timer tied to the strength of gravity so that the character moves slightly but not so much that it launches off the elevator. After soliciting responses and tinkering with this design, Raybeam shared the link to his level so readers could test it and offer more 
feedback. Raybeam's post, like others in the feedback-to-feedback section of the community, allows members to draw on knowledge about the community to improve their skills at design and making video games.

Members of Hogwarts at Ravelry also have a number of opportunities for learning, tinkering, and making available within their online community. One of the most central fixtures for hanging out and tinkering with tools for making at Hogwarts is its "house" system. Created in the image of the Harry Potter series, each house (Gryffindor, Ravenclaw, Hufflepuff, and Slytherin) has its own section of the forums for individualized support and feedback. In these houses, members can meet other participants and share and receive advice about projects. For example, one participant who goes by KnittingPrincipal was learning how to design her own knit squares. After sharing her interest, another member explained how to design them by providing an Excel spreadsheet she had used herself. The color-codes in each cell of the spreadsheet provided a template for how to make the desired design. In this way, players use digital tools, like an Excel spreadsheet and the online community, to create blueprints for physical objects in the form of knits. The blueprints are created and shared online but are used to create physical objects, and participants often take pictures of their made goods to be shared again online for rewards provided by the house system.

An example of tinkering in action can be seen through the formal classes offered in the Hogwarts at Ravelry community by more experienced knitters. In each house, members role-play as students, attend classes, and fulfill assignments. Members trade their resources for learning with their peers during study hall, and they also turn in quizzes provided by the instructor, follow deadlines and due dates, and earn points as part of the class activities. During the classes the members learn to use research and writing to complete assignments that teach valuable skills for creative production. Some classes encourage research assignments that require reading up on Harry Potter books and related fiction. In one class taught by KnittingPrincipal, she required students to research and craft something in the image of the ancient Royal Library of Alexandria and the Harry Potter Durmstrang Institute. One of her students, Myriam, researched the lore of Harry Potter and tied her research to a crafted item in the image of that story (see Figure 4). Students interact with classmates and tap the community knowledge base of patterns and tutorials to construct knits used as part of the assignment. In another class taught by Nalia, students were required to learn about the Celtic Ogham runic alphabet and tie it into their own crafting activity. The house system and related classes offered within the Hogwarts at Ravelry community are key sites of interaction and knowledge sharing that support tinkering with both physical knits as well as new media representations like blueprints for knit construction. These free classes are self-organized by community members and typically last six weeks. 


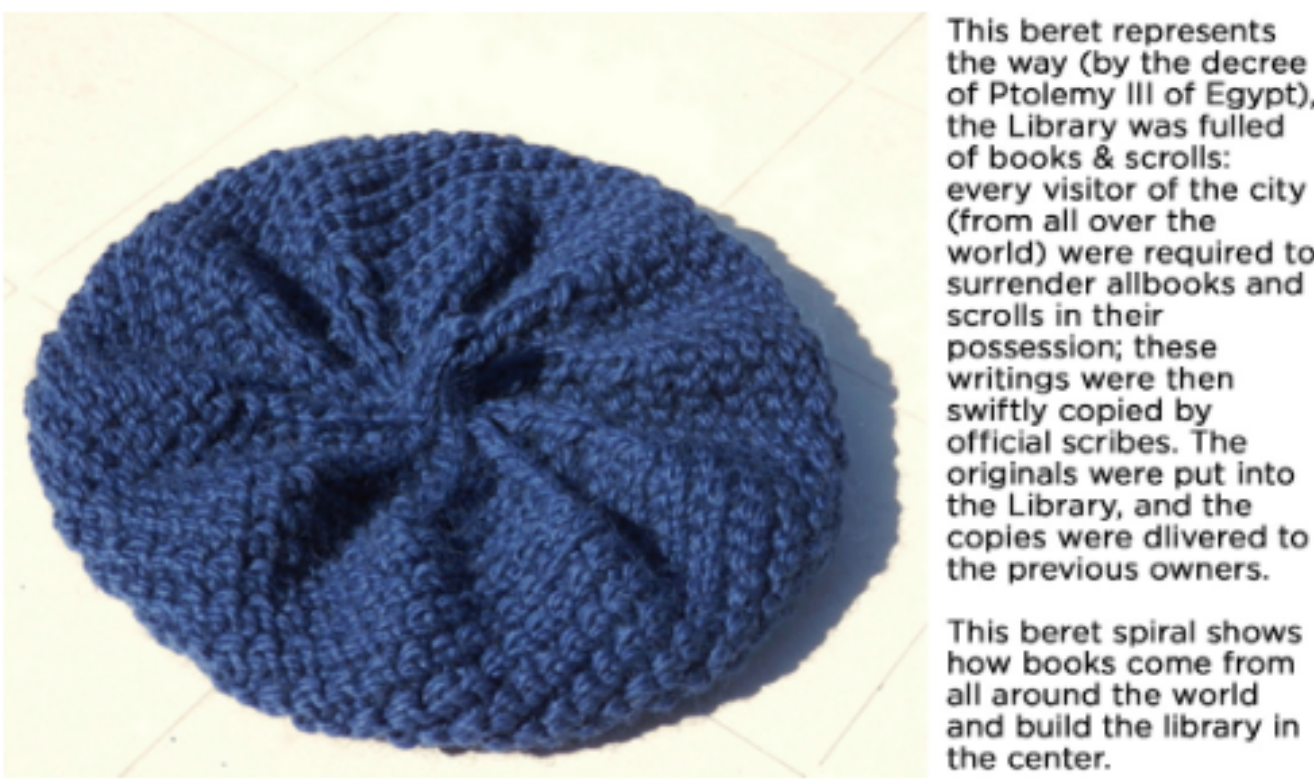

Figure 4: Myriam researched the Library of Alexandria, reported back on what she learned, and tied her research to a crafted item. Image courtesy of Myriam.

\section{Making and Online Communities: Learner Stories}

In previous sections, I described the openly networked design features of Sackboy Planet and Hogwarts at Ravelry, and then I discussed how these two cases develop a learning architecture within their respective communities to provide numerous opportunities for participants to pursue skill-building activities. In what follows, I share two examples that illustrate the learner trajectory more fully - how youth and young adults tinker online and the use openly networked features of their online communities to connect their work across a broader learning ecology.

Chris (14 years old), also known as Gadget 42 on Sackboy Planet, is a $10^{\text {th }}$ grader in the United States who has passions in theater, English literature, and gaming. He started playing the LBP2 video game through a friend who introduced him to it, and he became "hooked" ever since. His initial pursuits involved messing around with the ingame level editor that allows players to not only play the side-scrolling adventure game but also create new video games for others to play, as well. "I liked it right away. I would build little cars...it was fairly simple at first but when I started to try more advanced and it got more difficult." As Chris started taking on more of these difficult challenges, he searched the Internet for tutorials and found the Sackboy Planet community. "The people [on Sackboy Planet] were friendly...we all have something in common, which is LittleBigPlanet." His initial forays in the community were posts where he asked questions about design in order to get help from more experienced participants. Over time, as he received feedback and improved his own skills at game design, he began to offer help to others who posted questions. For Chris, the Sackboy Planet community enriched the potential of the game and enabled him to improve his skills as a designer among a supportive group of peers. Sackboy Planet provides a connected learning 
environment centered on making online goods in the form of participant-created video games.

Like other Sackboy Planet members we interviewed, Chris found connections between his own academic interests in English literature and the design activities he pursues in the game and online among peers. As an English student, he writes stories for class that he also uses to help think of ideas for level design projects. "I learn to develop a story arc, which I then use for LittleBigPlanet to create the beginning, middle, and end of the story level I'm making. Currently I am writing and making a murder mystery pointand-click adventure game." For Chris, his English literature skills overlap well with level design. To produce effective level designs he mixes both computer programming with story development to create the murder mystery.

Sackboy Planet was also where Chris made new friends who became fellow gamers and collaborative tinkerers as budding level designers. After hanging out with others in the forums and in the chatrooms, he began to build closer relationships with others who challenged his thinking around design: "It's nice seeing other people's views about the game." Every day he hangs out with friends online, talks about their shared interest, and helps others with design puzzles. "Most of the time I'm helping out friends. Trying to figure out the [design] logic for LBP2 and story and gameplay and stuff like that." In order to design levels using the game's level editor, participants like Chris must tap the knowledge base the Sackboy Planet community has assembled to improve their skills. Participants often share their level designs in forums embedded in the community, and fellow community members will play their draft levels and provide extensive feedback on how to improve it. Chris also uses tutorials that other community members develop that provide step-by-step guides for tweaking and tinkering with features of their own designs. Ultimately, his pursuits in the game and participation on Sackboy Planet among a supportive group of peers enabled him to mess around and tinker with level designing and develop the needed skills in logic and programming for online creative production.

Thanh is a 20-year-old knitter who lives in Vietnam and a participant of Hogwarts at Ravelry. She has interests in knitting but feels as though her local friends think it is silly and joke about it. She thinks that DIY activities like fiber crafting are on the rise for her generation, but until the interest gains more traction locally she for now pursues her interests online to find communities of support. After searching for knitting guides online, she found Ravelry and encountered many other people who share her interests in knitting. Her favorite group within the platform is a knitting challenge group, where participants sign up and are then assigned a challenge project tailored to their existing work. 'I've done one challenge and though I don't really like the final product, I do feel included in something communal, and that I successfully conquered a challenge." Thanh appreciates the experience of pursuing a challenge among peers with a shared interest.

As a member of Hogwarts at Ravelry, Thanh mostly watches the group's activities but also really likes to pick through the completed knitting projects that other users post to their online profile pages. "I learned mainly via jealous XD [emoticon for joking]. Just kidding. I mean, looking at others' projects, especially those with intarsia, fair isle, entrelac, complicated cabling, or nupps, is enough to push myself to learn more." Looking through other projects inspired and motivated Thanh to learn more techniques and improve her knitting. Before finding Hogwarts at Ravelry, Thanh's only 
experience with lace knitting was a scarf that involved two basic stitches. After joining the online community, she found and started a new lace project that required a new stich technique called slip-slip-knit (ssk). Learning this technique allowed her to create many related types of stitches and broaden the complexity of the projects she could produce. Moreover, when she encounters errors in her own work, she can look through the pattern pages of others' projects to find people who solved similar problems. The resources available and the supportive community at Hogwarts at Ravelry allowed Thanh to pursue her interest beyond the constraints of her local environment and learn and develop skills as a fiber crafter.

\section{Discussion}

Although research on making, digital media, and learning have identified how online communities can provide opportunities for youth and young adults to "hang out," "mess around," and "geek out" as they pursue making activities, little work explores how making practices translate beyond the confines of these learning communities (Ito et al. 2009; 2013). As reported in this chapter, participants of online communities learn to develop skills necessary to craft made goods, both physical and digital, through interactions online. These interactions take the form of peer-supported feedback and mentorship, as well as access to peer-created resources like tutorials and other guides. These online spaces are thus important as learning resources in that they provide not simply blueprints for learning but also a rich social environment for interaction that connects learners to various opportunities.

An empirically underexplored feature of connected learning theory is how the openly networked design of online platforms can productively connect makerspaces to other spheres of engagement across the learning ecology of participants. Openly networked design refers to the capacity of online platforms and digital tools that can render learning resources plentiful and broadly accessible (Ito et al. 2013). This chapter demonstrates not only the existence of these features embedded in each community, but how participants variably take them up to extend their work into other important parts of their lives. Sackboy Planet participants use the easily linkable database of their creations to share their work in other settings, like Twitter, to extend their reach and connect with fans and friends in another online setting. Hogwarts at Ravelry makers uniquely distribute their interest into other online networks both online and offline. In the case of Hogwarts at Ravelry, participants fluidly move back and forth between knitting and construction of tangible objects, creating digital representations of those artifacts online to share and circulate, and then move back into creating tangible objects as a result of feedback and opportunities created within the Hogwarts at Ravelry online community. Such movement across openly networked design features effectively bridges multiple arenas across the learning ecology and provides many different ways to share their work and engage with others.

The interconnectedness of making, particularly as supported by online platforms for learning and sharing, is an important site of inquiry for research in this area. Infrastructures that facilitate sharing knowledge and made artifacts allow participants to render their interest pursuits visible across multiple settings and provide new means 
through which to make their work meaningful beyond the confines of any given community of practice (Ito et al. 2013).

Still unexplored, however, is how mentors, both within the learning community and in other parts of the learning ecology around youth makers, shape how maker practices are not only transposed to other spheres but rendered meaningful to these other spaces. Mentors have consistently been noted as a key mechanism to the development of skills within a community of practice, but not necessarily as nodes that productively connect young people's work to other spheres (Jenkins et al. 2009). For example, Hogwarts at Ravelry provides an architecture for learning by not only making accessible various instructional materials to learners but by connecting them to more experienced knitters who teach the classes. Similarly, new Sackboy Planet participants are actively identified by more experienced members of the community and provided opportunities to share and receive feedback as part of their development. This chapter illustrates how environments cultivate learners and makers. Mapping out the many facets of students' learning ecologies, and expanding the scope to online communities where many youth and young adults inhabit, is key to identifying the means to support connected learning pursuits for all learners. 


\section{References}

Barron, Brigid. 2006. "Interest and Self-Sustained Learning as Catalysts of Development: A Learning Ecology Perspective." Human Development 49(4):193-224.

Black, Rebecca W. 2008. Adolescents and online fan fiction. Vol. 23. Peter Lang.

Bronfenbrenner, U. 1979. The Ecology of Human Development. Cambridge, MA: Harvard University Press.

Eisenberg, M., \& Buechley, L. 2008. "Pervasive fabrication: Making construction ubiquitous in education." Journal of Software, 3(4), 62-68.

Gee, James P. 2003. "What Video Games Have to Teach Us About Literacy and Learning." Computers in Entertainment 1(1):1-4.

Guzzetti, B., Elliott, K., \& Welsch D. 2010. DIY Media in the classroom: New literacies across content areas. New York, NY: Teachers College Press.

Horst, Heather A, Becky Herr-Stephenson, and Laura Robinson. 2010. "Media Ecologies." Pp. 29-78 in Hanging Out, Messing Around, and Geeking Out: Kids Living and Learning with New Media. Cambridge, MA: MIT Press.

Ito, Mizuko et al. 2009. Hanging Out, Messing Around, and Geeking Out: Kids Living and Learning with New Media. Cambridge, MA: MIT Press.

Ito, Mizuko, Daisuke Okabe, and Izumi Tsuji. 2012. Fandom Unbound: Otaku Culture in a Connected World. New Haven, CT: Yale University Press.

Ito, M., Gutiérrez, K., Livingstone, S., Penuel, B., Rhodes, J., Salen, K., Schor, J., SeftonGreen, S., and Watkins, S.C. 2013. Connected Learning: An Agenda for Research and Design. Irvine, CA: Digital Media and Learning Research Hub.

Jenkins, H. 2006. Convergence culture: Where old and new media collide. New York: New York University Press.

Jenkins, Henry, Katie Clinton, Ravi Puruchotma, Alice J Robinson, and Margaret Weigel. 2009. Confronting the Challenges of Participatory Culture: Media Education for the 21st Century. Cambridge, MA: MIT Press.

Korobkova, Ksenia A. 2014. Schooling the Directioners: Connected Learning and Identity-Making in the One Direction Fandom. Irvine, CA: Digital Media and Learning Research Hub.

Kow, Yong Ming, Timothy Young, and Katie Salen Tekinbaş. 2014. Crafting the Metagame: Connected Learning in the StarCraft II Community. Irvine, CA: Digital Media and Learning Research Hub.

Lankshear, C., \& Knobel, M. 2010. DIY Media: Creating, sharing and learning with new technologies (New literacies and digital epistemologies). New York, NY: Peter Lang.

Liebowitz, S J, and Stephen E Margolis. 1994. "Network Externality: An Uncommon Tragedy." The Journal of Economic Perspectives 8(2):133-150.

Martin, Crystle. 2014. Learning the Ropes: Connected Learning in a WWE Fan Community. Irvine, CA: Digital Media and Learning Research Hub.

Pfister, Rachel Cody. 2014. Hats for House Elves: Connected Learning and Civic Engagement in Hogwarts at Ravelry. Irvine, CA: Digital Media and Learning Research Hub.

Rafalow, Matthew H., and Katie Salen Tekinbaş. 2014. Welcome to Sackboy Planet: Connected Learning Among LittleBigPlanet 2. Irvine, CA: Digital Media and 
Learning Research Hub.

Spencer, A. 2005. DIY: The rise of lo-fi culture. London, England: Marion Boyars. Wortham, S. 2011. Youth cultures, language, and literacy. Review of Research in Education 35:vii-xi. 